\title{
Combination therapy of an IL-15 superagonist complex, ALT-803, and a tumor targeting monoclonal antibody promotes direct antitumor activity and protective vaccinal effect in a syngenic mouse melanoma model
}

\author{
Xiaoyue Chen ${ }^{1}$, Bai Liu', Kaiping Han ${ }^{1}$, Lin Kong ${ }^{1}$, Terra Noel ${ }^{1}$, Emily K Jeng², Sarah Alter ${ }^{*}$, Mark Rubinstein ${ }^{3}$, \\ Peter R Rhode', Hing C Wong ${ }^{2}$
}

From 30th Annual Meeting and Associated Programs of the Society for Immunotherapy of Cancer (SITC 2015) National Harbor, MD, USA. 4-8 November 2015

Cytokine-based and antibody-targeted immunotherapies have both been important approaches in the treatment of malignant cancers. However, combinational therapies of cytokines and tumor-targeting antibodies remain to be further explored, especially in advanced solid tumors. In this study, C57BL/6 mice bearing established subcutaneous B16F10 melanoma were treated with mouse melanoma targeting anti-gp75 monoclonal antibody (mAb), TA99, combined with interleukin (IL)-15 based superagonist ALT-803. This soluble protein complex consists of an IL-15 superagonist mutant (IL-15N72D) associated with an IL-15 receptor $\alpha$ Sushi domain human IgG1 Fc fusion protein. Compared to native IL15, ALT-803 possesses superior in vivo biologic activity for stimulating $\mathrm{NK}$ and $\mathrm{CD} 8^{+}$memory $\mathrm{T}$ cells. The combined ALT-803+TA99 therapy significantly exceeded either monotherapy in inhibiting melanoma tumor growth $(\mathrm{p}<0.001)$ and prolonging survival $(\mathrm{p}<$ 0.01 ) of B16F10 tumor-bearing mice. Through immune cell depletion studies and immunophenotyping of peripheral cells as well as tumor-infiltrating leukocyte subsets, we found that ALT-803 enhances TA99-mediated antitumor immunity through activation of NK cells and expansion of the $\mathrm{CD} 8^{+} \mathrm{CD} 44^{\text {high }}$ memory $\mathrm{T}$ cell arm. In contrast, $\mathrm{CD}^{+} \mathrm{T}$ cells were shown to play more of a suppressive role in the therapeutic effect of ALT-803 +TA99, possibly through involvement of regulatory

${ }^{1}$ Altor Bioscience, Miramar, FL, USA

Full list of author information is available at the end of the article
$\mathrm{T}$ cells or ALT-803-mediated induction of PD-L1 on $\mathrm{CD}_{4}^{+} \mathrm{T}$ cells in the periphery and tumor microenvironment. Addition of anti-PD-L1 mAb to ALT-803+TA99 therapy resulted in a further increase in antitumor activity against subcutaneous B16F10 tumors. Furthermore, tumor-bearing mice that survived due to ALT-803 +TA99 combination therapy exhibited long term antitumor memory against B16F10 tumor cell rechallenge. Immune-depletion studies revealed that this antitumor memory was associated with $\mathrm{CD}_{4}^{+} \mathrm{T}$ cells, $\mathrm{CD}^{+} \mathrm{T}$ cells and NK cells. Our findings suggest a therapeutic opportunity for ALT-803 in combination with tumor-targeting antibodies to simultaneously augment targeted antitumor activities of therapeutic antibodies and induce a long-term vaccinal effect which will provide durable responses in the treated host.

\footnotetext{
Authors' details

${ }^{1}$ Altor Bioscience, Miramar, FL, USA. ${ }^{2}$ Altor BioScience Corporation, Miramar, FL, USA. ${ }^{3}$ Medical University of South Carolina, Charleston, SC, USA.

Published: 4 November 2015

doi:10.1186/2051-1426-3-S2-P347

Cite this article as: Chen et al:: Combination therapy of an IL-15 superagonist complex, ALT-803, and a tumor targeting monoclonal antibody promotes direct antitumor activity and protective vaccinal effect in a syngenic mouse melanoma model. Journal for ImmunoTherapy of Cancer 2015 3(Suppl 2):P347.
} 